\title{
Robust, Semi-Intelligible Isabelle Proofs from ATP Proofs
}

\author{
Steffen Juilf Smolka and Jasmin Christian Blanchette \\ Technische Universität München, Germany
}

\begin{abstract}
Sledgehammer integrates external automatic theorem provers (ATPs) in the Isabelle/HOL proof assistant. To guard against bugs, ATP proofs must be reconstructed in Isabelle. Reconstructing complex proofs involves translating them to detailed Isabelle proof texts, using suitable proof methods to justify the inferences. This has been attempted before with little success, but we have addressed the main issues: Sledgehammer now transforms the proofs by contradiction into direct proofs (as described in a companion paper [4]); it reconstructs skolemization inferences; it provides the right amount of type annotations to ensure formulas are parsed correctly without overwhelming them with types; and it iteratively tests and compresses the output, resulting in simpler and faster proofs.
\end{abstract}

\section{Introduction}

Sledgehammer [22] is a proof tool that connects the Isabelle/HOL proof assistant [19] with external automatic theorem provers (ATPs), including first-order resolution provers and SMT solvers. Given an interactive proof goal, it heuristically selects hundreds of facts (lemmas, definitions, and axioms) from Isabelle's vast libraries, translates them to first-order logic (FOL), and invokes the external provers. Although Sledgehammer can be trusted as an oracle, ${ }^{1}$ most users are satisfied only once the proof has been reduced to Isabelle primitives.

When Sledgehammer was originally conceived, the plan was to have it deliver detailed proofs in Isabelle's Isar language [31], a textual, human-readable format inspired by Mizar [17]. Paulson and Susanto [23] designed a prototype that performs inference-by-inference translation of ATP proofs into Isar proofs and justifies each Isar inference using metis, a proof method based on Hurd's Metis resolution prover [14]. This idea was abandoned for several reasons: The resulting proofs by contradiction were unpalatable, so that users were disinclined to insert them in their theory text; they were often syntactically incorrect due to technical issues; and a single metis call with the short list of needed lemmas usually sufficed to re-find the proof.

Proof reconstruction with metis one-liners means that the proof must be re-found each time the Isabelle theory text is processed. This sometimes fails for difficult proofs that metis cannot re-find within a reasonable time and is vulnerable to small changes in the formalization. It also provides no answer to users who would like to understand the proof-whether it be novices who expect to learn from it, experts who must satisfy their curiosity, or merely skeptics. But perhaps more importantly, metis supports no theories beyond equality, which is becoming a bottleneck as automatic provers are being extended with dedicated procedures for theory

\footnotetext{
${ }^{1}$ For many years, Sledgehammer employed type-unsound encodings by default [18], making it unsuitable as an oracle. Newer versions use optimized type-sound encodings [5].
} 
reasoning. The Z3-based smt proof method [7] is a powerful alternative to metis, but it depends on the availability of Z3 on the user's machine for proof replay, which hinders its acceptance among users. Moreover, due to its incomplete quantifier handling, it can fail to re-find a proof generated by a resolution prover.

The remedy to all these issues is well known: to generate detailed, structured Isar proofs based on the machine-generated proofs, as originally envisioned by Paulson and Susanto. The first issue is that the Isar proof, like the underlying ATP proof, is by contradiction. A companion paper describes an algorithm that turns such proofs around [4]. The present paper describes further enhancements that increase the intelligibility and robustness of the output and that are implemented in Sledgehammer's proof translation pipeline (Section 3).

- Skolemization: Sledgehammer communicates with ATPs in full FOL as opposed to quantifier-free clause normal form (CNF). Skolemization is performed by the external ATPs, but it must be reconstructed in Isar (Section 4).

- Type annotations: Isabelle can generate strings from formulas, but it does not always understand its own output. Terms are often read back with overly general polymorphic types, resulting in failures. Annotating each subterm with type constraints impedes readability. Instead, Sledgehammer now employs an algorithm that introduces a minimal, complete set of type annotations (Section 5).

- Proof preplay: Sledgehammer users waste precious time on proofs that fail or take too long. Proof preplay addresses this by testing the generated proofs for a few seconds before presenting them to users. If several proofs are available, users can choose the fastest one and insert it in their theory text (Section 6).

- Proof compression: The generated proofs can be arbitrarily detailed depending on which ATP is used. Users normally want to compress straightforward chains of deduction into single Isar inferences, justified by a single metis call. This is performed in combination with preplaying to obtain faster and simpler proofs (Section 7).

Although the focus is on Isabelle, most of these techniques are equally applicable to proof construction for other Sledgehammer-like tools, such as HOL(y)Hammer for HOL Light [15] and MizAR for Mizar [1].

\section{Isabelle/HOL}

The Isabelle/HOL proof assistant is based on polymorphic higher-order logic (HOL) [11] extended with axiomatic type classes [30]. The types and terms of HOL are that of the simplytyped $\lambda$-calculus [9] augmented with type constructors, type variables, and term constants.

The types are either type variables (e.g., $\alpha, \beta)$ or $n$-ary type constructors, usually written in postfix notation (e.g, $\alpha$ list). Nullary type constructors are also called type constants (e.g., nat). The binary type constructor $\alpha \rightarrow \beta$ is interpreted as the (total) function space from $\alpha$ to $\beta$. Type variables can carry type class constraints, which are essentially predicates on the types. 
Terms are either constants (e.g., map), variables (e.g., $x$ ), function applications (e.g., $f x$ ), or $\lambda$-abstractions (e.g., $\lambda x . f x x$ ). Constants and variables can be functions. HOL formulas are simply terms of type bool. The familiar connectives and quantifiers are predefined $(\neg, \wedge, \vee$, $\longrightarrow, \forall, \exists)$. Constants can be polymorphic; for example, map : $(\alpha \rightarrow \beta) \rightarrow \alpha$ list $\rightarrow \beta$ list applies a unary function elementwise to a list of $\alpha$ elements.

Isabelle is a generic theorem prover whose metalogic is an intuitionistic fragment of HOL. In the metalogic, propositions have type prop, universal quantification is written $\wedge$, implication is written $\Longrightarrow$, and equality is written $\equiv$. The object logic is embedded in the metalogic using a constant Trueprop : bool $\rightarrow$ prop, which is normally not printed. Some foundational properties can only be expressed in the metalogic, but they play no role in Sledgehammer. We preserve the distinction between the two levels to avoid distracting the trained Isabelle eye, but readers unfamiliar with Isabelle can safely ignore the distinction.

Types are inferred using Hindley-Milner inference. Type annotations : $\tau$ give rise to additional constraints that further restrict the inferred types. A classic example where type annotations are needed is $2+2=4$. Without type annotations, the formula is parsed as $(2: \alpha)+(2: \alpha)=(4: \alpha)$, where $\alpha$ belongs to the numeric type class, which defines basic numeric operators and syntax but imposes no semantics on the "numbers." An annotation is necessary to make the formula provable-e.g., $(2: i n t)+2=4$. A single annotation is sufficient because of the constraints arising from the most general types of the involved operators: op $+: \alpha \rightarrow \alpha \rightarrow \alpha$ and op $=: \alpha \rightarrow \alpha \rightarrow$ bool.

For both types and terms, Isabelle distinguishes two kinds of free variable: schematic variables, which can be instantiated, and nonschematic variables, which stand for fixed, unknown entities. When stating a conjecture and proving it, the type and term variables are normally fixed, and once it is proved, they become schematic so that users of the lemma can instantiate them when applying the lemma.

\section{The Translation Pipeline}

The translation from an ATP proof to an Isar proof involves two main intermediate data structures. The ATP proof is first parsed and translated into a proof by contradiction with the same structure but with HOL formulas instead of first-order formulas. The proof is then transformed into a direct proof, from which Isar proof text is synthesized. Various operations are implemented on these data structures to enhance the proof.

ATP Proof. Paulson and Susanto had the foresight to choose TSTP (Thousands of Solutions for Theorem Provers) [28] as input format for their prototype. Among the automatic provers they wanted to integrate with Isabelle, only E [25] supported the format at the time. Nowadays, most provers feature some support for TSTP.

TSTP specifies the basic syntax for representing proofs as a directed acyclic graph of inferences. A single parser can be used to integrate all provers that can generate the syntax. However, the format does not mandate any proof system; hence, interfacing a new ATP usu- 
ally requires some work, especially for processing inferences that introduce new symbols (e.g., skolemization). Isar proof construction is currently supported for the resolution provers $\mathrm{E}$ and Vampire [24] and the unit-equality prover Waldmeister [13]. For the future, we are also interested in the higher-order provers LEO-II [2] and Satallax [8], which are partly integrated in Sledgehammer already [26].

SPASS generates proofs in its custom DFG format only (even though it can parse TPTP FOF [27]). Fortunately, DFG is based on similar concepts and can be represented using the same data structure as TSTP in memory, so it is also supported to a large extent.

Proof by Contradiction. The ATP proof is translated into an Isabelle proof by contradiction. This step preserves the graph structure of the proof, but the nodes are labeled by HOL formulas.

Some consolidation can already take place at this level. ATPs tend to record many more inferences than are interesting to Isabelle users. For example, trivial operations such as clausification and variable renaming produce linear inference chains that can be collapsed.

This translation corresponds largely to the work by Paulson and Susanto. We refer to their paper [23] for details. In particular, they describe how HOL terms, types, and type classes are reconstructed from their encoded FOL form. Their code had to be adapted to cope with the variety of type encodings supported by newer versions of Sledgehammer [5], but nonetheless their description fairly accurately describes the current state of affairs.

Direct Proof. The proof redirection algorithm, presented in the companion paper [4], takes a proof by contradiction as the input and produces a direct proof. The latter can be regarded as a fragment of Isar proofs. The abstract syntax of proofs $(\pi)$ and inferences $(\iota)$ is given by the production rules

$$
\begin{aligned}
\pi & :=\left(\text { fix } x^{*}\right)^{*}(\text { assume } l: \phi)^{*} \iota^{*} \\
\iota::=\operatorname{prove} q^{*} l: \phi l^{*} \pi^{*} & \\
& \mid \text { obtain } q^{*} x^{*} \text { where } l: \phi l^{*} \pi^{*}
\end{aligned}
$$

where $x$ ranges over HOL variables (which may be of function types), $\phi$ over HOL formulas, $l$ over Isar fact labels (names), and $q$ over Isar qualifiers (then and show). Asterisks $\left(^{*}\right.$ ) denote repetition. Nested proof blocks are possible, as indicated by the syntax $\pi^{*}$.

A fix command fixes the specified variables in the local context, and assume enriches the context with an assumption. Standard inferences are performed using prove. Its variant obtain proves the existence of HOL variables for which a property holds; the variables are added to the context.

Once the direct proof is constructed, it is iteratively compressed and preplayed. Finally, qualifiers are introduced: then indicates that the previous fact is needed to prove the current fact, whereas show is required for the last inference in the top-level block. The then keyword is only a convenience; the same effect can be achieved less elegantly using labels. At the end, useless labels are removed, and the remaining labels are changed to $f 1, f 2$, etc. 
Isar Proof. The final step of the translation pipeline produces a textual Isar proof. This step is straightforward, but some care is needed to generate strings that can be parsed back by Isabelle. This is especially an issue for formulas, where type annotations might be needed.

Example. The following Isabelle theory fragment declares a two-valued state datatype, defines a flip function, and states a conjecture about flip:

$$
\begin{aligned}
& \text { datatype state }=\text { On } \mid \text { Off } \\
& \text { fun flip : state } \rightarrow \text { state where } \\
& \text { flip On }=\text { Off } \mid \\
& \text { flip Off }=\text { On } \\
& \text { lemma flip } x \neq x
\end{aligned}
$$

\begin{tabular}{|c|c|c|c|c|}
\hline 51 & axiom & flip(on) $=$ off & \multicolumn{2}{|c|}{ flip_simps_1 } \\
\hline 52 & axiom & flip $($ off $)=$ on & \multicolumn{2}{|c|}{ flip_simps_2 } \\
\hline 55 & axiom & $\neg$ off $=$ on & \multicolumn{2}{|c|}{ state_distinct_1 } \\
\hline 57 & axiom & $\forall X_{3}\left(\neg \operatorname{state}\left(X_{3}\right)=\right.$ on $\longrightarrow \operatorname{state}\left(X_{3}\right)=$ off $)$ & \multicolumn{2}{|c|}{ state_exhaust } \\
\hline 58 & axiom & $\operatorname{state}(s)=s$ & \multicolumn{2}{|c|}{ type_of_s } \\
\hline 774 & conj & $\neg \mathrm{flip}(\mathrm{s})=\mathrm{s}$ & \multicolumn{2}{|l|}{ goal } \\
\hline 775 & neg_conj & $\neg \neg \mathrm{flip}(\mathrm{s})=\mathrm{s}$ & 774 & negate \\
\hline 776 & neg_conj & $f l i p(s)=s$ & 775 & flatten \\
\hline 781 & plain & off $\neq$ on & 55 & flatten \\
\hline 892 & plain & $\forall X_{0}\left(\neg \operatorname{state}\left(X_{0}\right)=\right.$ on $\longrightarrow \operatorname{state}\left(X_{0}\right)=$ off $)$ & 57 & rectify \\
\hline 893 & plain & $\forall X_{0}\left(\right.$ state $\left(X_{0}\right) \neq$ on $\longrightarrow \operatorname{state}\left(X_{0}\right)=$ off $)$ & 892 & flatten \\
\hline 1596 & plain & $\forall X_{0}\left(\right.$ state $\left(X_{0}\right)=$ on $\vee \operatorname{state}\left(X_{0}\right)=$ off $)$ & 893 & ennf_trans \\
\hline 2238 & neg_conj & $f l i p(s)=s$ & 776 & cnf_trans \\
\hline 2239 & plain & state $(s)=s$ & 58 & cnf_trans \\
\hline 2287 & plain & flip(on) $=$ off & 51 & cnf_trans \\
\hline 2288 & plain & flip $($ off $)=$ on & 52 & cnf_trans \\
\hline 2375 & plain & off $\neq$ on & 781 & cnf_trans \\
\hline 2485 & plain & $\forall X_{0}\left(\right.$ state $\left(X_{0}\right)=$ off $\vee \operatorname{state}\left(X_{0}\right)=$ on $)$ & 1596 & cnf_trans \\
\hline 3342 & plain & on $=\mathrm{s} \vee \operatorname{state}(\mathrm{s})=$ off & 2239,2485 & superpos \\
\hline 3362 & plain & on $=s \vee$ off $=s$ & 3342,2239 & fwd_demod \\
\hline 3402 & neg_conj & $\mathrm{flip}(\mathrm{on})=\mathrm{on} \vee$ off $=\mathrm{s}$ & 2238,3362 & superpos \\
\hline 3404 & neg_conj & off $=$ on $\vee$ off $=s$ & 3402,2287 & fwd_demod \\
\hline 3405 & neg_conj & $\mathrm{off}=\mathrm{s}$ & 3404,2375 & subsum_res \\
\hline 3407 & neg_conj & flip $($ off $)=$ off & 3405,2238 & bwd_demod \\
\hline 3408 & neg_conj & off $=$ on & 3407,2288 & fwd_demod \\
\hline 3409 & neg_conj & $\perp$ & 3408,2375 & subsum_res \\
\hline
\end{tabular}

Invoking Sledgehammer launches a collection of ATPs (typically, E, SPASS, Vampire, and Z3). The conjecture is easy, so they rapidly return. Vampire delivers the following proof, presented in a slightly abbreviated TSTP-like format: 
The formulas used from the original problem are listed first. Each line gives a formula number, a role, and a FOL formula. Any problem formula that can be used to prove the conjecture is an axiom for the automatic prover, irrespective of its status in Isabelle (lemma, definition, or actual axiom). The rightmost columns indicate how the formulas was arrived at: Either it appeared in the original problem, in which case its identifier is given (e.g., flip_simps_1), or it was derived from one or more already proved formulas using a Vampire-specific proof rule.

If Sledgehammer's isar_proofs option is enabled, textual Isar proof reconstruction is attempted. The Isabelle proof by contradiction for the ATP proof above is as follows:

$\begin{array}{rll}775 & \text { flip } s=s & \neg \text { goal } \\ 3402 & \text { flip On }=\text { On } \vee \text { Off }=s & 775, \text { state.exhaust } \\ 3404 & \text { Off }=\text { On } \vee \text { Off }=s & 3402, \text { flip.simps }(1) \\ 3405 & \text { Off }=s & 3404, \text { state.distinct(1) } \\ 3407 & \text { flip Off }=\text { Off } & 775,3405 \\ 3409 & \text { False } & 3407, \text { flip.simps(2), state.distinct }(1)\end{array}$

Linear inference chains are drastically compressed, and the lemmas

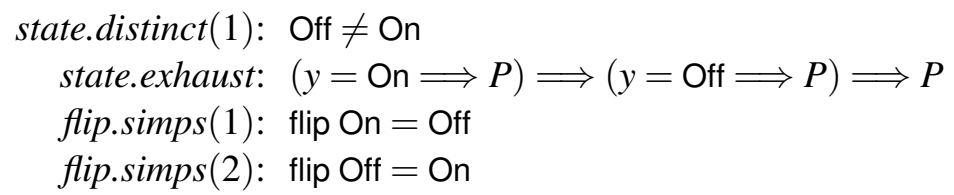

are referenced by name rather than repeated. The passage from FOL to HOL also eliminates encoded type information, such as the state function and the auxiliary axiom type_of_s. After redirection, the proof becomes

$$
\begin{aligned}
& \text { prove [] 3407: “flip Off } \neq \text { Off” }[\text { flip.simps(2), state.distinct }(1)][] \\
& \text { prove [] 3405: “flip } s \neq s \vee \text { Off } \neq s[3407][] \\
& \text { prove [] 3404: “flip } s \neq s \vee \text { Off } \neq s \wedge \text { Off } \neq \text { On }[3405, \text { state.distinct }(1)][] \\
& \text { prove [] 3402: “flip } s \neq s \vee \text { flip On } \neq \text { On } \wedge \text { Off } \neq s[3404, \text { flip.simps(1)] [] } \\
& \text { prove [show] 775: "flip } s \neq s "[3402, \text { state.exhaust] [] }
\end{aligned}
$$

Compression and cleanup simplify the proof further:

$$
\begin{aligned}
& \text { prove [] } \epsilon \text { : “flip Off } \neq \text { Off" }[\text { flip.simps(2), state.distinct }(1)][] \\
& \text { prove [then, show] } \epsilon: \text { : flip } s \neq s "[\text { flip.simps }(1), \text { state.distinct }(1), \text { state.exhaust }][]
\end{aligned}
$$

From this simplified direct proof, the Isar proof is easy to produce:

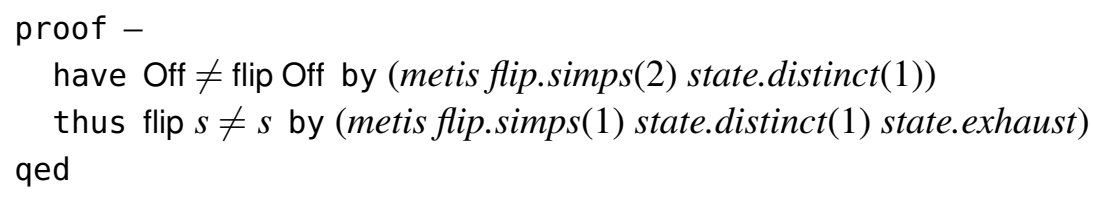




\section{Skolemization}

The typical architecture of modern first-order provers combines a clausifier and a CNF-based reasoning core. It is the clausifier's duty to skolemize the problem and move the nonskolemizable quantifiers to the front of the formulas, where they can be omitted. Sledgehammer historically performed clausification itself, using a naive exponential application of distributive laws. This was changed a few years ago to use the ATPs' native clausifiers, which generate a polynomial number of clauses [3, §6.6.1]. Skolemization transforms a formula into an equisatisfiable, but not equivalent, formula. As a result, it must be treated specially when reconstructing the proof. Simply invoking metis, as done in Paulson and Susanto's prototype, will not work to replay skolemization inferences.

Conjecture and axioms are treated differently because of their different polarities. By convention, the axioms are positive and the conjecture is negative. ${ }^{2}$ In the positive case, skolemization eliminates the essentially existential quantifiers (i.e., the positive occurrences of $\exists$ and the negative occurrences of $\forall$ ). In the negative case, it eliminates the essentially universal quantifiers. Negative skolemization is usually called dual skolemization or herbrandization [12].

E and Vampire explicitly record skolemization inferences in their proof, and fortunately they do it in the same way. On the other hand, SPASS's proofs are expressed in terms of the clausified problem; we have some ideas on how to recover the missing information but have yet to try them out.

The Positive Case. We start with the easier, positive case. Consider the following concrete but archetypal extract from an E or Vampire proof:

$$
\begin{array}{llll}
11 & \text { axiom } & \forall X \exists Y \mathrm{p}(X, Y) & \text { exists_P } \\
53 & \text { plain } & \forall X \mathrm{p}(X, \mathrm{y}(X)) & 11 \quad \text { skolem }
\end{array}
$$

In Isar, a similar effect is achieved using the obtain command:

$$
\text { obtain } y \text { where } \forall x \text {. P } x(y x) \text { by (metis exists_P) }
$$

In the abstract Isar-like data structure that stores direct proofs, the inference is represented as

$$
\text { obtain [] [y] where 53: “ } \forall x \text {. P } x(y x) "[\text { exists_P] [] }
$$

The approach works for arbitrary quantifier prefixes. All essentially existential variables are eliminated simultaneously. For example, the ATP proof fragment

$$
\begin{aligned}
& 18 \text { axiom } \forall V \exists W \forall X \exists Y \forall Z \mathrm{q}(V, W, X, Y, Z) \quad \text { exists_Q } \\
& 90 \text { plain } \forall V \forall X \forall Z \mathrm{q}(V, \mathrm{w}(V), X, \mathrm{y}(V, X), Z) 18 \text { skolem }
\end{aligned}
$$

is translated to

\footnotetext{
${ }^{2}$ This choice is justifiable from the point of view of an automatic prover that attempts to derive $\perp$ from a set of axioms and a negated conjecture, because all the premises it starts from and the formulas it derives are then considered positive.
} 
obtain [] [w,y] where 90: “ $\forall v x z \cdot \mathrm{Q} v(w v) x(y v x) z$ " [exists_Q] []

Reconstruction crucially depends not only on metis's clausifier but also on its support for mildly higher-order problems, because of the implicit existential quantification over the Skolem function symbols in obtain. Indeed, metis is powerful enough to prove a weak form of the HOL axiom of choice:

lemma $(\forall x . \exists y . P x y) \Longrightarrow \exists f . \forall x . P x(f x)$

by metis

Of course, nothing is derived ex nihilo: metis can only prove the formula because its clausifier depends on the axiom of choice in the first place. Furthermore, metis will succeed only if its clausifier puts the arguments to the Skolem functions in the same order as in the proof text. This is not difficult to ensure in practice: Both $\mathrm{E}$ and metis respect the order in which the universal variables are bound, whereas Vampire uses the opposite order, which is easy to reverse.

Positive skolemization suffers from a technical limitation connected to polymorphism. Lemmas containing polymorphic skolemizable variables cannot be reconstructed, because the variables introduced by obtain must have a ground type. An easy workaround would be to relaunch Sledgehammer with a monomorphizing type encoding [5, §3] to obtain a more suitable ATP proof. A more challenging alternative would involve detecting which monomorphic instances of the problematic lemmas are needed and re-engineer the proof accordingly.

The Negative Case. In the ATPs, negative skolemization of the conjecture is simply reduced to positive skolemization of the negated conjecture. For example:

$$
\begin{array}{lllll}
25 & \text { conj } & \forall V \exists W \forall X \exists Y \forall Z \mathrm{q}(V, W, X, Y, Z) & \multicolumn{2}{l}{\text { goal }} \\
41 & \text { neg_conj } & \neg \forall V \exists W \forall X \exists Y \forall Z \mathrm{q}(V, W, X, Y, Z) & 25 & \text { negate } \\
43 & \text { neg_conj } & \neg \exists W \exists Y \mathrm{q}(\mathrm{v}, W, \mathrm{x}(W), Y, \mathrm{z}(W, Y)) & 41 & \text { skolem }
\end{array}
$$

However, once the proof has been turned around in Sledgehammer, the last two lines are unnegated and exchanged: First, a proof of the (unnegated) conjecture is found for specific fixed variables (cf. formula 43 above); then these are generalized into quantified variables (cf. formula 41). A natural name for this process is un-herbrandization. In Isar, the fix command achieves a similar effect, as in the example below:

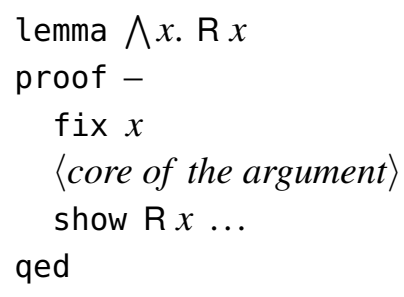

However, this works only for the outermost universal quantifiers. Since we cannot expect users to always state their conjectures in this format, we must generally use a nested proof block, enclosed in curly braces. Thus, the ATP proof fragment presented above is translated to 


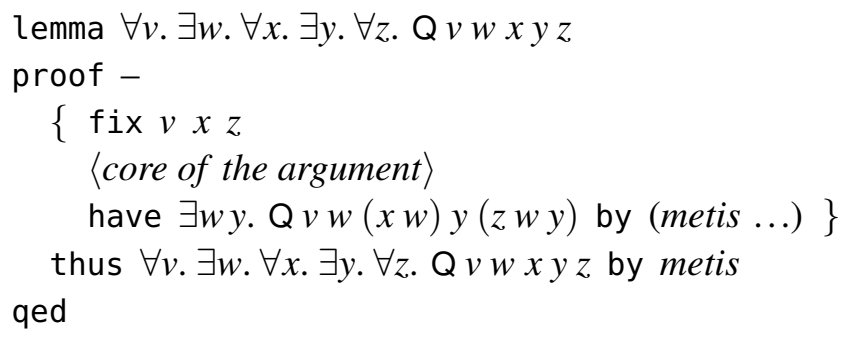

Seen from outside, the nested block proves the formula $\bigwedge v x z . \exists w y . Q v w(x w) y(z w y)$. From there, metis derives the desired formula $\forall v . \exists w . \forall x . \exists y . \forall z . Q v w x y z$, in which the quantifiers alternate arbitrarily. In the data structure that stores direct Isar-like proofs, the proof would be represented as

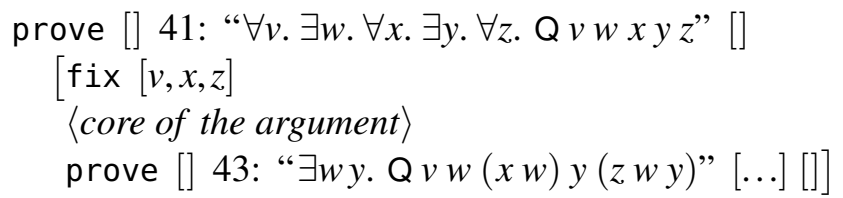

An easy optimization, which is not yet implemented, would be to omit the nested proof block for conjectures of the form $\bigwedge x_{1} \ldots x_{n} . \phi$, where $\phi$ contains no essentially universal quantifiers. It should also be possible to move the inferences that do not depend on the herbrandized symbols outside the nested block.

Alternative Approaches. Given a HOL problem, the metis method clausifies it and translates it to FOL, invokes the first-order prover Metis, and replays the Metis inferences using suitable HOL tactics. Skolemization is simulated using Hilbert's choice operator $\varepsilon$ [23]; for example, $\forall x$. $\exists y$. P $x y$ is skolemized into $\forall x$. P $x$ ( $\varepsilon y$. P $x y$ ). A newer experimental skolemizer exploits Isabelle's schematic variables to eliminate the dependency on Hilbert's choice [3, §6.6.7], only requiring the weak axiom of choice to move the existentials to the front. Whichever approach is used, Sledgehammer's textual proof construction exploits metis's machinery (and the reduction of HOL to FOL) instead of replicating it textually.

Other ATP-based proof methods or tactics must also cope with skolemization. Isabelle's smt method [7] relies on Hilbert's choice, whereas HOL(y)Hammer's proof reconstructor [15] depends only on the weak axiom of choice. Another option is to trust the ATP's clausifier, leaving it to the user to inspect the generated clausification axioms; this is the approach imple-

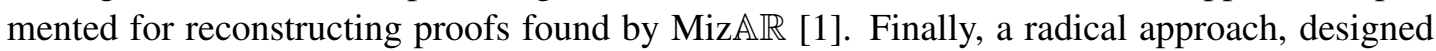
for textual proof reconstruction in Coq, is to replace Skolem function symbols by predicate symbols and adjust the proof accordingly, a process known as deskolemization [10].

\section{Type Annotations}

To ensure that types are inferred correctly when the generated HOL formulas are parsed again by Isabelle, it is necessary to introduce type annotations. However, redundant annotations 
should be avoided: If we insisted on annotating each subterm, the simple equation $x s=y s$, where $x s$ and ys range over lists of integers, would be rendered as

$$
((\text { op }=: \text { int list } \rightarrow \text { int list } \rightarrow \text { bool })(x s: \text { int list }): \text { int list } \rightarrow \text { bool })(y s: \text { int list }): \text { bool }
$$

The goal is not to make the Hindley-Milner inference redundant but rather to guide it.

Paulson and Susanto's prototype generates no type annotations at all. Isabelle provides alternative print modes (e.g., one mode annotates all bound variables at the binding site) but none of them is complete. This may seem surprising to users familiar with other proof assistants, but Isabelle's extremely flexible syntax, combined with type classes, means that some terms cannot be parsed back.

We implemented a custom "print mode" for Sledgehammer, which might become an official Isabelle mode in a future release. The underlying algorithm computes a locally minimal set of type annotations for a formula and inserts the annotations. In Isabelle, type annotations are represented by a polymorphic constant $a_{\tau}: \tau \rightarrow \tau$ that can be thought of as the identity function. The term ann $\tau t$ is printed as $t: \tau$. In the presentation below, the notation $t^{\tau}$ indicates that term $t$ has type $\tau$.

The Algorithm. Given a well-typed formula $\phi$ to annotate, the algorithm starts by replacing all the types in $\phi$ by the special placeholder_ (Isabelle's "dummy" type). It then infers the most general types for $\phi$ using Hindley-Milner, resulting in a formula $\phi^{\star}$ in which the placeholders are instantiated. Next, it computes the substitution $\rho=\left\{\alpha_{1} \mapsto \tau_{1}, \ldots, \alpha_{m} \mapsto \tau_{m}\right\}$ such that $\phi^{\star} \rho=\phi$, which must exists if $\phi$ is well-typed and the inferred types in $\phi^{\star}$ are the most general. Finally, the algorithm inserts type annotations of the form : $\tau$ that cover all the type variables $\alpha_{i}$ in $\rho$ 's domain-i.e., such that each type variable $\alpha_{i}$ occurs in at least one type annotation.

The last step is where the complexity arises. The algorithm assigns a cost to each candidate site $t^{\tau}$ in $\phi$ where a type annotation can be inserted. The cost is given as a triple of numbers:

$$
\text { cost of } t^{\tau}=(\text { size of } \tau \text {, size of } t \text {, preorder index of } t \text { in } \phi)
$$

Triples are compared lexicographically. The first two components encode a preference for smaller annotations and smaller annotated terms. The third component resolves ties by preferring annotations occurring closer to the beginning of the printed formula. All subterms of $\phi$ are potential candidates to carry type annotations. (It would be desirable to consider the binding sites of variables in quantifiers and $\lambda$-abstractions as candidates as well, but unfortunately these are simply name-type pairs and not terms in Isabelle.) Each site $t^{\tau}$ is also associated with the set of type variables $\alpha_{i}$ it covers.

The goal is to compute a minimal set of sites that completely covers all type variables. The resulting cost need not be a global minimum, though; computing the minimum amounts to solving the weighted set cover problem, which is NP-hard [16]. One could probably use a SAT solver to solve the problem efficiently, but we prefer a more direct greedy approach, which is polynomial and produces satisfactory results in practice. 
Starting with the set of all possible sites, the algorithm iteratively removes the most expensive redundant site until the set is minimal in the sense that removing any site from it would make it incomplete. This reverse greedy approach ensures that a minimal set will be reached eventually. In contrast, the classical greedy approach could yield a too large set: For the term $\mathrm{h}^{\text {nat } \rightarrow \text { real }} \mathrm{c}^{\text {nat }}$ generalized to $\mathrm{h}^{\alpha \rightarrow \beta} \mathrm{c}^{\alpha}$, it would first pick $\mathrm{c}$ to cover $\alpha$, only to find out that $\mathrm{h}$ must be annotated as well to cover $\beta$, making the first site redundant.

The names of the variables $\alpha_{i}$ introduced in $\phi^{\star}$ are irrelevant as long as they are fresh. In a postprocessing step, variables that occurs only once as a subtype in $\tau_{1}, \ldots, \tau_{m}$ are replaced by _, and annotations : $\tau$ that cover only variables converted to _ are omitted. Thus, the formula length $([]: \alpha$ list $)=0$ is printed as length []$=0$ without undesirable gain of generality.

Example. Let fst : $\alpha \times \beta \rightarrow \alpha$ and snd : $\alpha \times \beta \rightarrow \beta$ be polymorphic constants that extract the components of a pair. Suppose the formula $\phi$ to annotate is

$$
\forall x y . \exists p \text {. fst } p=x \wedge \text { snd } p=y
$$

with $x$ : nat and $y$ : real. Inside Isabelle, the formula's subterms carry type information (except the bound variables):

$$
\begin{aligned}
& \text { All }^{(\text {nat } \rightarrow \text { bool }) \rightarrow \text { bool }}\left(\lambda x ^ { \text { nat } } \text { . All } { } ^ { ( \text { real } \rightarrow \text { bool } ) \rightarrow b o o l } \left(\lambda y ^ { \text { real } } \text { . Ex } { } ^ { ( \text { nat } \times \text { real } \rightarrow \text { bool } ) \rightarrow b o o l } \left(\lambda p^{\text {nat } \times \text { real }}\right.\right.\right. \text {. } \\
& (\mathrm{op} \vee)^{\text {bool } \rightarrow \text { bool } \rightarrow \text { bool }}\left((\mathrm{op}=)^{\text {nat } \rightarrow \text { nat } \rightarrow \text { bool }}\left(\mathrm{fst}^{\text {nat } \times \text { real } \rightarrow \text { nat }} p\right) x\right) \\
& \left.\left.\left.\left((\text { op }=)^{\text {real } \rightarrow \text { real } \rightarrow \text { bool }}\left(\text { snd }^{\text {nat } \times \text { real } \rightarrow \text { real }} p\right) y\right)\right)\right)\right)
\end{aligned}
$$

Replacing the types with _ yields the formula

$$
\mathrm{All}^{-}\left(\lambda x^{-} . \mathrm{All}^{-}\left(\lambda y^{-} . \mathrm{Ex}^{-}\left(\lambda p^{-} .(\mathrm{op} \vee)^{-}\left((\mathrm{op}=)^{-}\left(\mathrm{fst}^{-} p\right) x\right)\left((\mathrm{op}=)^{-}\left(\mathrm{snd}^{-} p\right) y\right)\right)\right)\right)
$$

from which type inference produces the formula $\phi^{\star}$ :

$$
\begin{gathered}
\text { All }{ }^{(\alpha \rightarrow b o o l) \rightarrow \text { bool }}\left(\lambda x ^ { \alpha } \text { . All } { } ^ { ( \beta \rightarrow b o o l ) \rightarrow b o o l } \left(\lambda y^{\beta} . \mathrm{Ex}^{(\alpha \times \beta \rightarrow b o o l) \rightarrow \text { bool }}\left(\lambda p^{\alpha \times \beta} .\right.\right.\right. \\
(\mathrm{op} \vee)^{\text {bool } \rightarrow \text { bool } \rightarrow \text { bool }}\left((\mathrm{op}=)^{\alpha \rightarrow \alpha \rightarrow b o o l}\left(\mathrm{fst}^{\alpha \times \beta \rightarrow \alpha} p\right) x\right) \\
\left.\left.\left.\left((\mathrm{op}=)^{\beta \rightarrow \beta \rightarrow \text { bool }}\left(\text { snd }^{\alpha \times \beta \rightarrow \beta} p\right) y\right)\right)\right)\right)
\end{gathered}
$$

The substitution entailed by $\phi$ and $\phi^{\star}$ is $\rho=\{\alpha \mapsto n a t, \beta \mapsto$ real $\}$. There are several possible ways to annotate the formula so as to cover both $\alpha$ and $\beta$, including

$$
\begin{aligned}
& \forall x y . \exists p . \text { fst }(p: \text { nat } \times \text { real })=x \wedge \text { snd } p=y \\
& \forall x y . \exists p .(\text { fst } p: \text { nat })=x \wedge(\text { snd } p: \text { real })=y \\
& \forall x y . \exists p . \text { fst } p=(x: \text { nat }) \wedge \text { snd } p=(y: \text { real })
\end{aligned}
$$

The third formula is the one produced by the reverse greedy algorithm. It is arguably the most aesthetically pleasing of the three, because both the annotated terms and the types are atomic.

Incidentally, the annotations could have been omitted in this example because the property holds generally for arbitrary types $\alpha$ and $\beta$, but this cannot always be relied upon. Moreover, omitting the type annotations is not completely harmless because of the poor interaction between skolemization and polymorphism. 


\section{Proof Preplay}

Isar proofs generated from ATP proofs sometimes fail. We already mentioned that skolemization is not supported for polymorphic variables (cf. Section 4). The TSTP or DFG parser occasionally goes wrong if it encounters unexpected (undocumented) syntax. The ATP proof can also contain inferences that are ill-typed from an Isabelle point of view-despite the use of globally sound encodings, individual inferences can violate the type discipline. Moreover, the proof reconstruction code is not bug-free. And even in the absence of errors, the Isar proofs can fail because metis fails to discharge a proof obligation within a reasonable amount of time.

As the experience with Sledgehammer-generated metis one-liners has shown, it is advantageous to try out, or preplay, the proofs before presenting them to users [3, §6.6.6]. The proofs are then printed together with timing information, including warnings about timeouts or failures. Since Sledgehammer invokes multiple ATPs in parallel, users must often choose between several one-liners and structured Isar proofs. Based on the preplay information, they can make an informed decision while being spared the tedium of having to test the proofs manually.

Until recently, users had to enable Isar proof reconstruction to benefit from it. The preplay mechanism makes a new hybrid mode possible, in which an Isar proof is generated whenever the one-liner times out or fails. Even when the Isar proof is not entirely correct, it is easier to repair than the corresponding monolithic one-liner-for example, by adding some parentheses to guide the parsing or by replacing a failing or too slow metis call by a hand-written proof.

The following example, based on an Isabelle formalization of the Robbins conjecture [29], demonstrates preplaying. When invoked on the goal $x \sqcup-x=-x \sqcup--x$ and configured to use Waldmeister, Sledgehammer produces the following output:

Try this: by (metis huntington sup_assoc sup_comm) (> $3 \mathrm{~s})$.

Structured proof (54 steps, $1.33 \mathrm{~s})$ :

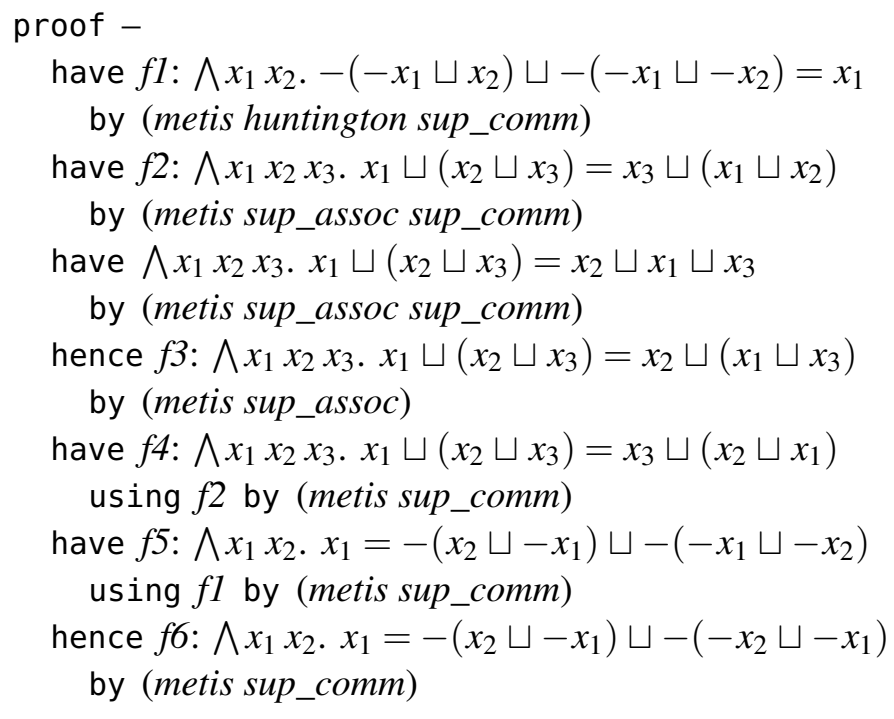




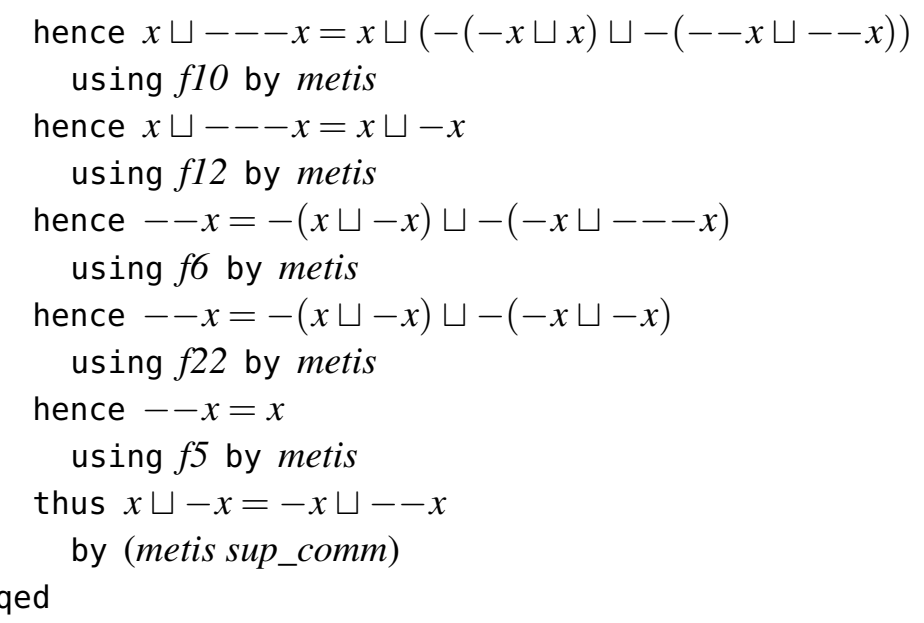

Waldmeister found a proof involving the same three lemmas over and over (huntington, sup assoc, and sup_comm). However, metis fails to re-find the proof within 3 seconds, as indicated by the mention " $>3 \mathrm{~s}$ " on the first line. (Indeed, metis stands no chance even if given several minutes.) In contrast, the above (abridged) 54-step Isar proof was replayed in 1.33 seconds. Users can click it to insert it in their proof text and move on to the next conjecture.

Behind the scenes, the Isar proof preplay procedure starts by enriching the context with all the local facts introduced in the proof $(f 1, f 2$, etc.). For each inference $\Phi \vdash \phi$, it measures the time metis takes to deduce $\phi$ from $\Phi$ and stores it in a data structure. The total is printed at the end, with a '>' prefix if any of the metis calls timed out. In the rare event that a metis call failed prematurely, Sledgehammer displays the mention "may fail" in the banner.

An alternative approach would have been to have Isabelle parse the Isar proof using its usual interfaces, thereby covering more potential sources of error. For example, with our approach the Isabelle terms are not printed and reparsed; because of Isabelle's flexible syntax, parsing is problematic even if enough type annotations are inserted. On the other hand, the better coverage would come at the price of additional overhead, and it is not clear how to achieve it technically. More importantly, the alternative approach offers no way to collect timing information on a per-step basis. This information is essential for proof compression, as we will see in the next section, and recomputing it would waste the user's time.

Currently, metis is invoked to reconstruct each ATP inference in Isabelle. With proof preplay in place, it should be easy to try out other proof methods. To reconstruct proofs with theory-specific or higher-order reasoning, we would need both to appeal to existing decision procedures in Isabelle (e.g., for linear arithmetic) and develop dedicated methods.

\section{Proof Compression}

The generated Isar proofs can involve dozens or hundreds of steps. It is usually beneficial to compress them. Compressed proofs can be faster to recheck; for example, when the Robbins proof from Section 6 is compressed from 54 to 29 steps, Isabelle also takes nearly half a second 
less to process it. Moreover, many users prefer concise Isar proofs, either because they want to avoid cluttering their theory files or because they find the shorter proofs simpler to understand. Of course, compression can also be harmful: A metis one-liner is nothing but an Isar proof compressed to the extreme, and it can be both very slow and very cryptic.

Whereas intelligibility is in the eye of the beholder, speed can be measured precisely via preplay. Our compression procedure considers candidate pairs of inferences and performs the merger if the resulting inference is fast enough-no more than $20 \%$ slower than the original inferences taken together. This $20 \%$ tolerance factor embodies a trade-off between processing speed and conciseness. Given the inferences $\Phi_{1} \vdash \phi_{1}$ and $\left\{\phi_{1}\right\} \uplus \Phi_{2} \vdash \phi_{2}$, where $\phi_{1}$ is not referenced elsewhere in the proof (in an antecedent), the merged inference is $\Phi_{1} \cup \Phi_{2} \vdash \phi_{2}$.

The algorithm consists of the following steps:

1. Initialize the worklist with all inferences $\Phi \vdash \phi$ such that $\phi$ is referenced only once in the rest of the proof.

2. If the worklist is empty, stop; otherwise, take an inference $\Phi_{1} \vdash \phi_{1}$ from the worklist.

3. Let $\left\{\phi_{1}\right\} \uplus \Phi_{2} \vdash \phi_{2}$ be the unique inference that references $\phi_{1}$. Try to merge the two inferences as described above. If this succeeds, add any emerging singly-referenced facts belonging to $\Phi_{1} \cap \Phi_{2}$ to the worklist.

\section{Go to step 2 .}

Step 2 nondeterministically picks an inference. Our implementation prefers inferences with long formulas, because these clutter the proof more. In step 3, merging the two inferences may give rise to new singly-referenced facts $\phi$ that were referenced by both $\phi_{1}$ and $\phi_{2}$ (i.e., $\phi \in \Phi_{1} \cap \Phi_{2}$ ) but not by any other inferences.

The process is guided by metis's performance. Users who want to understand the proof may find that too many details have been optimized away. For them, there is a Sledgehammer option that controls the compression factor, which bounds the number of mergers before the algorithm stops in relation to the length of the uncompressed proof.

\section{Conclusion}

The latest version of Sledgehammer employs a variety of techniques to improve the readability and efficiency of the generated Isar proofs. Whenever one-line proof reconstruction fails or times out, users are offered detailed, direct Isar proofs that discharge the goal, sometimes after a small amount of manual tuning. Users who are interested in inspecting the proofs can force their generation by passing an option. Related options control preplay and compression.

This work is still in progress. Many aspects could be improved further; we mentioned a few in the previous sections. Our next priority is to identify and rectify any remaining failure cases: Preplaying insulates users from failures, but ideally valid ATP proofs should always lead to valid Isar proofs. We plan to integrate the proof reconstruction code with the "Judgment Day" harness [6] to test it more thoroughly and evaluate its impact on Sledgehammer's success rate. 
The next step will be to implement proof manipulation algorithms to simplify the proofs further before presenting them to users. For example, users normally prefer sequential chains of deduction to the spaghetti-like structure of some machine-generated proofs; using appropriate algorithms, it should be possible to minimize the number of jumps or introduce block structure to separate independent subproofs. Similar work has been carried out for human-written proofs [20, 21], but we expect machine proofs to offer more opportunities for refactoring.

Acknowledgment. Tobias Nipkow, Lawrence Paulson, Geoff Sutcliffe, and Josef Urban encouraged us to pursue this research. Stefan Berghofer and Cezary Kaliszyk shared ideas with us on how to address the problem of inserting type annotations. Cezary Kaliszyk, Andrei Popescu, Mark Summerfield, and three anonymous reviewers provided helpful feedback on earlier versions of this paper. The second author's research was financed by the Deutsche Forschungsgemeinschaft (DFG) project Hardening the Hammer (grant Ni 491/14-1).

\section{References}

[1] J. Alama. Escape to Mizar from ATPs. In P. Fontaine, R. Schmidt, and S. Schulz, editors, PAAR2012, pages 3-11, 2012.

[2] C. Benzmüller, L. Paulson, F. Theiss, and A. Fietzke. Progress report on LEO-II, an automatic theorem prover for higher-order logic. In K. Schneider and J. Brandt, editors, TPHOLs: Emerging Trends. C.S. Dept., Technische Universität Kaiserslautern, 2007.

[3] J. C. Blanchette. Automatic Proofs and Refutations for Higher-Order Logic. Ph.D. thesis, Dept. of Informatics, Technische Universität München, 2012.

[4] J. C. Blanchette. Redirecting proofs by contradiction. In J. C. Blanchette and J. Urban, editors, PxTP 2013, 2013.

[5] J. C. Blanchette, S. Böhme, A. Popescu, and N. Smallbone. Encoding monomorphic and polymorphic types. In N. Piterman and S. Smolka, editors, TACAS 2013, volume 7795 of LNCS, pages 493-507. Springer, 2013.

[6] S. Böhme and T. Nipkow. Sledgehammer: Judgement Day. In J. Giesl and R. Hähnle, editors, IJCAR 2010, volume 6173 of LNAI, pages 107-121. Springer, 2010.

[7] S. Böhme and T. Weber. Fast LCF-style proof reconstruction for Z3. In M. Kaufmann and L. Paulson, editors, ITP 2010, volume 6172 of LNCS, pages 179-194. Springer, 2010.

[8] C. E. Brown. Satallax: An automatic higher-order prover. In B. Gramlich, D. Miller, and U. Sattler, editors, IJCAR 2012, volume 7364 of LNCS, pages 111-117. Springer, 2012.

[9] A. Church. A formulation of the simple theory of types. J. Symb. Log., 5(2):56-68, 1940.

[10] H. de Nivelle. Translation of resolution proofs into short first-order proofs without choice axioms. Inf. Comput., 199(1-2):24-54, 2005.

[11] M. J. C. Gordon and T. F. Melham, editors. Introduction to HOL: A Theorem Proving Environment for Higher Order Logic. Cambridge University Press, 1993.

[12] J. Herbrand. Thèses présentées à la Faculté des sciences de Paris pour obtenir le grade de docteur ès sciences mathématiques. Ph.D. thesis, Science Faculty, Université de Paris, 1930.

[13] T. Hillenbrand, A. Buch, R. Vogt, and B. Löchner. WALDMEISTER-High-performance equational deduction. J. Autom. Reasoning, 18(2):265-270, 1997. 
[14] J. Hurd. First-order proof tactics in higher-order logic theorem provers. In M. Archer, B. Di Vito, and C. Muñoz, editors, Design and Application of Strategies/Tactics in Higher Order Logics, number CP-2003-212448 in NASA Technical Reports, pages 56-68, 2003.

[15] C. Kaliszyk and J. Urban. PRocH: Proof reconstruction for HOL Light. In M. P. Bonacina, editor, $C A D E-24$, volume 7898 of LNAI, pages 267-273. Springer, 2013.

[16] R. M. Karp. Reducibility among combinatorial problems. In R. E. Miller and J. W. Thatcher, editors, Complexity of Computer Computations, IBM Research Symposia Series, pages 85-103. Plenum Press, 1972.

[17] R. Matuszewski and P. Rudnicki. Mizar: The first 30 years. Mechanized Mathematics and Its Applications, 4(1):3-24, 2005.

[18] J. Meng and L. C. Paulson. Translating higher-order clauses to first-order clauses. J. Autom. Reasoning, 40(1):35-60, 2008.

[19] T. Nipkow, L. C. Paulson, and M. Wenzel. Isabelle/HOL: A Proof Assistant for Higher-Order Logic, volume 2283 of LNCS. Springer, 2002.

[20] K. Pąk. The methods of improving and reorganizing natural deduction proofs. In MathUI10, 2010.

[21] K. Pąk. Methods of lemma extraction in natural deduction proofs. J. Autom. Reasoning, 50(2):217228, 2013.

[22] L. C. Paulson and J. C. Blanchette. Three years of experience with Sledgehammer, a practical link between automatic and interactive theorem provers. In G. Sutcliffe, E. Ternovska, and S. Schulz, editors, IWIL-2010, 2010.

[23] L. C. Paulson and K. W. Susanto. Source-level proof reconstruction for interactive theorem proving. In K. Schneider and J. Brandt, editors, TPHOLs 2007, volume 4732 of LNCS, pages 232-245. Springer, 2007.

[24] A. Riazanov and A. Voronkov. The design and implementation of Vampire. AI Comm., 15(2-3):91110, 2002.

[25] S. Schulz. System description: E 0.81. In D. Basin and M. Rusinowitch, editors, IJCAR 2004, volume 3097 of $L N A I$, pages 223-228. Springer, 2004.

[26] N. Sultana, J. C. Blanchette, and L. C. Paulson. LEO-II and Satallax on the Sledgehammer test bench. J. Applied Logic, 11(1):91-102, 2013.

[27] G. Sutcliffe. The TPTP problem library and associated infrastructure-The FOF and CNF parts, v3.5.0. J. Autom. Reasoning, 43(4):337-362, 2009.

[28] G. Sutcliffe, J. Zimmer, and S. Schulz. TSTP data-exchange formats for automated theorem proving tools. In W. Zhang and V. Sorge, editors, Distributed Constraint Problem Solving and Reasoning in Multi-Agent Systems, volume 112 of Frontiers in Artificial Intelligence and Applications, pages 201-215. IOS Press, 2004.

[29] M. Wampler-Doty. A complete proof of the Robbins conjecture. In G. Klein, T. Nipkow, and L. Paulson, editors, The Archive of Formal Proofs. http://afp.sf. net/entries/Robbins Conjecture.shtml, 2010.

[30] M. Wenzel. Type classes and overloading in higher-order logic. In E. L. Gunter and A. Felty, editors, TPHOLs 1997, volume 1275 of LNCS, pages 307-322. Springer, 1997.

[31] M. Wenzel. Isabelle/Isar-A generic framework for human-readable proof documents. In R. Matuszewski and A. Zalewska, editors, From Insight to Proof-Festschrift in Honour of Andrzej Trybulec, volume 10(23) of Studies in Logic, Grammar and Rhetoric. University of Białystok, 2007. 